\title{
Genetic Algorithms for Portfolio Optimization with Weighted Sum Approach
}

\author{
Ricardo Faia ${ }^{1}$, Tiago Pinto ${ }^{2}$, Zita Vale ${ }^{1}$, Juan Manuel Corchado ${ }^{2}$ \\ ${ }^{1}$ GECAD Research Center, Polytechnic of Porto \\ Porto, Portugal \\ \{rfmfa, zav\}@isep.ipp.pt \\ ${ }^{2}$ BISITE Research Centre, University of Salamanca \\ Salamanca, Spain \\ \{tpinto, corchado\}@usal.es
}

\begin{abstract}
The use of metaheuristics to solve real-life problems has increased in recent years, since they are easy to implement, and the problems become easy to model when applying metaheuristic approaches. However, arguably the most important aspect is the execution time, since results can be obtained from metaheuristic methods in a much smaller time, and with a good approximation to the results obtained with exact methods. In this work, the Genetic Algorithm (GA) metaheuristic is adapted and applied to solve the optimization of electricity markets participation portfolios. This work considers a multiobjective model that incorporates the calculation of the return and the risk incurred in the electricity negotiations. Results of the proposed approach are compared to those achieved with an exact method, and it can be concluded that the proposed GA model is able to achieved very close results to those of the deterministic approach, in a much quicker execution time.
\end{abstract}

Keywords-Eletricity Markets, Genetic Algorithms, Metaheuristic, Portfolio Optimizaiton

\section{INTRODUCTION}

In many relevant optimization problems, the complexity of the problem and the computational constraints make it very difficult to find an exact solution within a reasonable time. In these cases, it is common practice to rely on the use of a heuristic optimization algorithm, that can find a good, maybe even the optimal, solution in a relatively short time.

Usually, in order to select suitable methods to optimize a problem, it is necessary for the user to have abundant information on the comparison of the possible methods, which in many cases the information available becomes insufficient. The choice of the user becomes even more difficult when the possible methods of resolution may be of different natures: for example, they may be stochastic or deterministic in nature. The stochastic methods offer a stochastic (probabilistic) guarantee of identifying the global solution, its theory states that the global solution will be found in an infinite time with a certain associated probability. On the other hand, by means of exact calculations the deterministic methods, after a finite (usually long) time, guarantee that an approximation of the global optimum will be found within the previously defined tolerances [1].

This work has received funding from the European Union's Horizon 2020 research and innovation programme under the Marie Sklodowska-Curie grant agreement No 641794 (project DREAM-GO) and grant agreement No 703689 (project ADAPT); and from FEDER Funds through COMPETE program and from National Funds through FCT under the project UID/EEA/00760/2013
Metaheuristic methods are currently an important part of global optimization algorithms, these algorithms are often inspired by nature with multiple interaction agents. A subset of metaheuristics can be called population based, in which we can highlight Genetic Algorithms (GA) Particle Swarm Optimization (PSO), both these algorithms are used frequently and produce good results, there are similarities and differences between both. GA is a population based algorithm, it solves problem maintaining a population of candidate solutions and creating new solutions by combining existing ones with usage of the crossover, mutation and selection operators, and keeping the candidate solution with the currently best fitness [2]. PSO solves a problem starting a population of candidate solution and moving these particles in the search domain according to the particles position and velocity. At each iteration, a particle of the swarm updates its position by following the best local particles and best solution of the whole swarm, thus guiding the swarm toward the best solutions [3]. Fig. 1 presents the generic metaheuristic search mechanism for all types of metaheuristics.

Metaheuristic approaches are often used because the programming of the problem is very flexible, easily a nonlinear problem can be solved, whereas the exact methods are extremely difficult to model the nonlinear functions. In Fig. 1 is presented the way in which, for example, a metaheuristic approach addresses non-linear functions whether they are constraints or objective functions. The process usually starts with the generation of an initial solution, then all the solutions of the set will be evaluated. The search process is then started, there is usually a stop criterion that defines when the algorithm stops searching. Until the search process finishes the algorithm iterations, at each iteration there is a search for a new solution, and in each iteration the solutions are evaluated, and the best remains until the end. When evaluating and validating the solutions in the metaheuristics if there is a nonlinear function, it is easy to perform this step, if the nonlinearities are mathematically formulated, it is enough to submit the generated solution and obtain the respective value. This step is expressed in orange in Fig. 1.

In this paper the GA metaheuristic is adapted and applied. GA is a highly parallel search and optimization technique within computational intelligence inspired in principle of Darwinian where the natural selection and genetic reproduction are natural 
techniques of human behavior and GA try to replicate in its implementation. The algorithm is implemented in a computational simulation, where a population of initial solutions is created to start the search for new and better solutions. Evolution usually starts from a randomly created solution and is run across generations. In each generation is performed the evaluation of population determining the fit or unfit individuals. Some individuals are selected for the next generation and then are combined with individuals previously selected or mutated to form a new population. This new population is input to the following iteration of the algorithm.

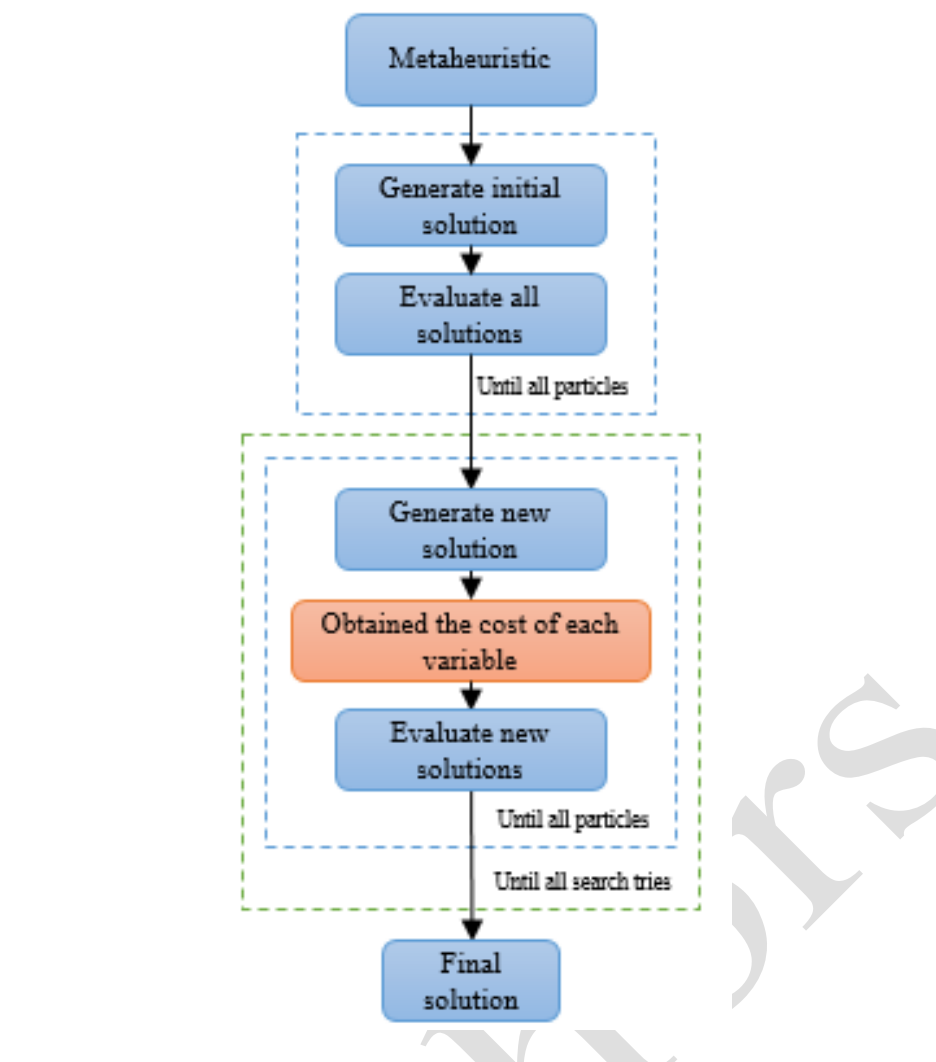

Fig. 1. Metaheuristic search mechanism

In this work the GA is applied to solve the portfolio optimization problem, applied to the electric power markets, which consists of the ideal allocation of electricity in the different markets in order to obtain the maximum return. The optimization is performed using MATLAB R2016a software.

This document is organized in five sections, where section I presents an introduction where the advantages of using the metaheuristics are analyzed and the most general ones are referenced. In section II a discussion is presented on the problem that is to be solved, where the most relevant state of art is described. In section III the methodology is described, the proposed GA method is explained, the formulation is presented and the case study is detailed. In section IV the results are presented and in $\mathrm{V}$ section the conclusions are presented.

\section{Portfolio OPtimizATION}

In the area of finance and economics, the portfolio optimization problem is used to study the allocation of funds among a range of risky assets, so that an objective function, usually obtained through a risk-adjusted return measure achieved is maximized. The work done by Markowitz has become innovative in laying the foundations of modern portfolio theory [4], this work has introduced the framework of the mean variance and justified that the ideal portfolio was determined based on the trade-off between maximizing the expected return and minimizing the risk.

In Electricity Markets (EM), the portfolio optimization has been used as a decision support tool, generally the markets of lettering are composed of different types of markets with different characteristics and time horizons. E.g. the day-ahead spot market allows for negotiation for the next day and bilateral contracts can be defined for a duration of years. This fact presents difficulties in the portfolio optimization, since the decisions for some trading can be less profitable in the future according to new information on prices. The consumers can take advantages when using a simulation with portfolio optimization, because there is a difference between signing a bilateral agreement with a convention generation plant or a solar photovoltaic power plant, a wind power plant, or a combination of any of these alternatives [5].

Kettunen et at [6], a study on contract portfolio optimization method, using multistage stochastic optimization, the study carried out by the author tends to give support to the retailer of electricity that is always obliged to fulfill a certain value of load often uncertain. In this work, the retailer will try to combine the electricity in the Spot market and can also carry out the hedge with the price of the futures markets, in order to later deliver its electricity. In the work is intended to minimize the cost associated with the purchase of electricity that will be subject to several restrictions of risk. The author considers that the retailer can purchase and sell forwards over multiple time periods depending on the evolution of the electricity prices and loads, thus calling the dynamic forward portfolio.

In the following work [7] GA is used to perform the portfolio optimization, in this case the problem is applied in a financial and economic area. The author uses different risk measures that are based on Markowitz's theory of Mean-Variance. In this work it is worth highlighting the applicability of the GA to the optimization problem of portfolios similar to what will be executed in this one.

In the traditional portfolio optimization, usually the considered risks come from the historical series analyzed, which are usually the historical prices of any asset [8]. In electricity markets this vision can also be applied and considered a multiobjective model where one will try to maximize profit and minimize risk. There are also different approaches where mathematical models are used that include uncertainty (risk), as is the case of stochastic models, where the price of the active (electricity) is not a single value, but a set of values, obtained through performance of the used forecasting methods [9]. 


\section{METHODOLOGY}

In this section we describe the GA metaheuristic that is used to solve the considered problem. The formulation of the portfolio optimization problem is also presented. The considered formulation is made up of two different objectives consisting of the profit from sales of electricity and the risk incurred by that sale. In order to make a resolution of the problem easier, we used a methodology called Weighted Sum Approach [10], which consists in transforming the multiobjective problem into a one objective with the inclusion of a weight factor. However, the task of selecting a set of weights that reflects preference to one goal or another can be difficult, because the preference tends to be the property of each user. In turn, an in-depth knowledge of the objectives and a balanced selection of weights may not make the final solution present the intended preferences that are supposed to be built into the weights. However, the resulting pareto front will depend on the weights that will be used, so it is important to determine how the weights relate to the references.

\section{A. Genetic Algorithms}

GA is a probabilistic technique for solving optimization problems based on population genetics, was introduced in the fist time by John Holland in the early 1970 years [11]. Normally, the method starts the search with a set of solutions called populations, each solution is represented by a chromosome, at each iteration the population size is preserved, in each iteration is executed the evaluation of each chromosome, for the next generation are probabilistically selected according to their fitness values. The chromosomes for the new generation can also be selected randomly, to produce new offspring, crossover and mutation occurs of the selected chromosomes. Since the chromosomes with the highest fitness value are more likely to be selected, the chromosomes of the new solution may have a higher average fitness value than those of the previous generation. The process of evolution is repeated until the final condition is satisfied [12]. The diagram shown in Fig. 2 depicts the AG screening methods as explained above.

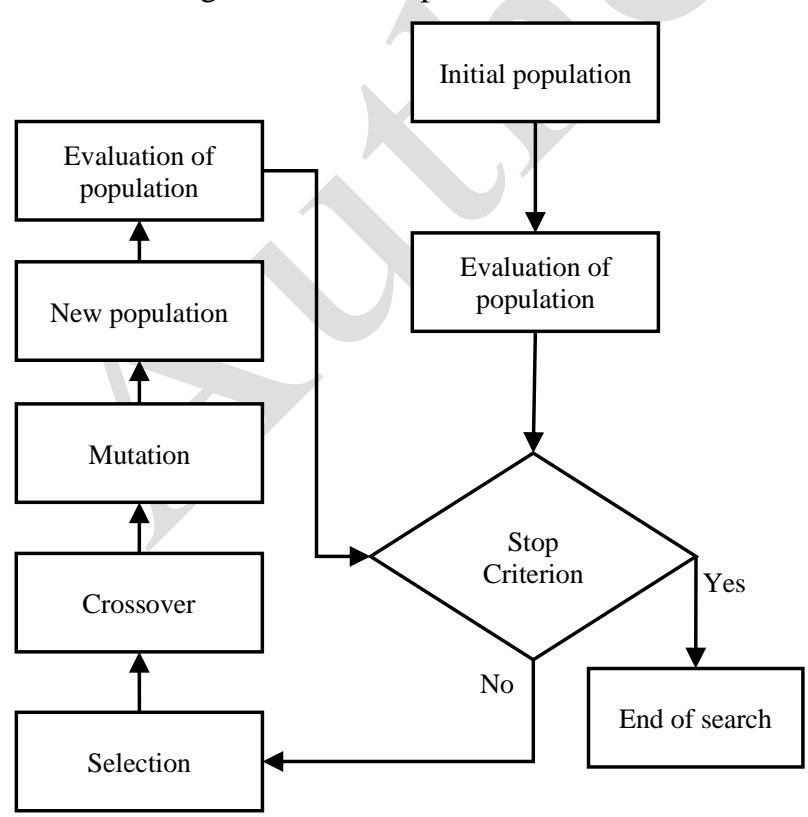

Fig. 2. GA flowchart [2]
As can be seen from the Fig. 2 the process starts with the generation of the initial solution that are randomly, population size depends on the nature of the problem, sometimes previous studies are carried out to obtain the ideal number for the population. Traditionally the solutions are randomly generated by the research space covering the entire range of possible solutions, in rare excesses the solutions can be placed in a particular research area to try to make the algorithm perform better. After this step the solutions will be evaluated, going to the next step in which the repetitive cycle begins until reaching the criterion of stop, if this is not verified the search goes to the selection step.

The selection is performed at each iteration, and a selected population proportion is selected, the selected solutions are usually selected by a process based on their fitness, where the best solutions are most likely to be selected. Some selection methods always select the best individuals in terms of fitness, others perform a random selection in the population, which can help maintain population diversity, which can avoid permutation in non-optimal solutions. The most popular solution methods are roulette wheel selection and tournament selection.

The next step according to the image is the process of crossover and mutation, which constitute the creation of the new generation, which is dependent on the individuals selected previously. To form a new solution a couple of solutions is selected for reproduction, coming from the group previously selected. In creating the new solution with crossover and mutation methods, this new solution shares many of the characteristics of the solutions that gave rise to it. This process ends when the number of solution created is equal to the number of solutions that existed previously. It is normal to have methods that use more than two parents to form the new solution. Usually on average the fitness of population is better in the new generation than in the previous one.

Once the process of creating the population is evaluated, it is evaluated through the objective function, and thus iteration ends, it is verified the criterion of stop if it is verified the algorithm returns the solution and ends the search. There are many stopping criteria that can be used, such as a fixed number of generations, allocated budget (computation time / money), the highest ranking solution and fitness, has reached a plateau such that successive iterations no longer produces better results.

\section{B. Formulation}

The presented methodology is characterized as a multiobjective problem where the two objectives are to maximize the profit and minimize the risk. The simplified problem formulation is presented in (1).

$$
\begin{aligned}
& \operatorname{Max}\left[\lambda \times\left(f 1\left(\text { Spow }_{\text {M...Nums }}, \text { Bpow }_{\text {M...NumM }}\right)\right)\right. \\
& \left.-(1-\lambda) \times\left(f 2\left(\operatorname{Ris}_{d, p}\right)\right)\right]
\end{aligned}
$$

where $f 1$ represents the return and $f 2$ the risk; $\lambda$ is the trade-off between profit and risk; $S p o w_{M}$ the volume to sell in market $M$; Bpow $_{M}$ volume to buy in session $S ; N u m M$ represents the number of considered markets; Risk ${ }_{d, p}$ represents the value of participation risk in $p$ period and $d$ day. 
Equation (2) represent the profit function, how can observe the first part represent the sales in different markets, the second part is the purchase in different markets, and the third the electricity production costs.

$$
\begin{gathered}
f 1\left(\text { Spow }_{M \ldots N u m M}, \text { Bpow }_{M \ldots N u m M}\right) \\
=\left[\begin{array}{c}
\sum_{M=M 1}^{N u m M}\left(\text { Spow }_{d, p, M} \times p S_{M, d, p} \times \text { Asell }_{M}\right)- \\
\sum \text { Cost }_{d, p}^{T E P}
\end{array}\right]
\end{gathered}
$$

where, Asell $_{M}$ and $A b u y_{M}$ represent the indication if this player can enter in negotiation in market $M$, and are boolean variables, Cost represents the function of production costs, TEP represents the total electricity produced, $S p o w_{d, p, M}$ represents the amount of electricity to sell in market $M, B p o w_{d, p, M}$ represents the amount of electricity to buy in market $M$ and $N u m M$ represents the number of markets.

Equation (3) represents the risk formulation, which is calculated based on the price forecasting error in each market, at each time. As can be observed, in both cases the risk is considered, both in the purchase as in the sale. The error values depending on the market where the trading is performed will have different levels of risk.

$$
\begin{aligned}
& f 2\left(\operatorname{Risk}_{d, p}\right) \\
& =\left[\sum_{M=M 1}^{N u m M}\left(\operatorname{Spow}_{M, d, p} \times p S_{M, d, p} \times \operatorname{error}_{M, d, p}\right)+\right]
\end{aligned}
$$

where, error $_{M, d, p}$ represent the error for market $M$, day $d$ and period $p$.

The next equation represents the considerations and constrains applied to the problem.

$$
\begin{aligned}
& \forall d \in N \text { day, } \forall p \in \text { Nper }, \text { Asell }_{M} \in\{0,1\}, \text { Abuy } \in\{0,1\}, \lambda \\
& \in\{0,1\} \\
& p S_{M, d, p} \\
& =\left\{\begin{array}{c}
\text { Forecast }_{M, d, p}^{A N N} \quad \text { if } M \notin \text { bilateral contacts } \\
\text { Value }\left(\operatorname{Spow}_{d, p, M}\right) \quad \text { if } M \in \text { bilateral contacts }
\end{array}\right. \\
& p B_{M, d, p} \\
& =\left\{\begin{array}{c}
\text { Forecast }_{M, d, p}^{A N N} \quad \text { if } M \notin \text { bilateral contacts } \\
\text { Value }\left(\text { Bpow }_{d, p, M}\right) \quad \text { if } M \in \text { bilateral contacts }
\end{array}\right. \\
& T E P=\sum \text { Energy }_{\text {prod }} \\
& \text { Energy }_{\text {prod }} \in\left\{\text { Renew }_{\text {prod }}, \text { Therm }_{\text {prod }}\right\} \\
& \operatorname{Cost}_{d, p}^{\text {TEP }}=a \times \text { Therm }_{\text {prod }}{ }^{2}+b \times \text { Therm }_{\text {prod }}+c
\end{aligned}
$$

where Forecast ${ }_{M, d, p}^{A N N}$ represents the price forecasting value of $A N N$ for market $M$, day $d$, and period $p$, Value $\left(\operatorname{Spow}_{d, p, M}\right)$, $\operatorname{Value}\left(\right.$ Bpow $\left._{d, p, M}\right)$ represents the values of price for amount electricity in bilateral contracts, Energy prod represents the electricity produced by the player, Renew $w_{\text {prod }}$ represents the renewable electricity produced, Therm prod $_{\text {represent the }}$ thermoelectric electricity produced and $a, b$ and $c$ represents the coefficients of marginal cost.

From equations (5) and (6) it can observed that two different types of prices are considered, the prices for bilateral contracts and prices from auction based markets. The origin of two different prices is caused by the characteristic of the markets, because in bilateral contract the price of electricity is highly variable depending on the amount of traded electricity, for estimation this values is used the clustering and fuzzy techniques, which can be seen in more detail in [13]. In other markets the electricity price is independent of the amount negotiated, this way an Artificial Neural Network is used [14]. The equation (7), (8) and (9) refers to the player production.

Equation (10) represents the main constrain of the problem, and represents the total electricity reserved to be sold in the set of all markets is never higher than the total expects production of the player, plus the bought electricity in all markets in the same period.

$$
\begin{gathered}
\sum_{M=M 1}^{N u m M} \text { Spow }_{M} \leq T E P+\sum_{M=M 1}^{N u m M} \text { Bpow }_{M} \\
0 \leq \text { Renew }_{\text {prod }} \leq \text { Max }_{\text {prod }} \\
\text { Min }_{\text {prod }} \leq \text { Therm }_{\text {prod }} \leq \text { Max }_{\text {prod }}, \text { if }_{\text {Therm }}^{\text {prod }}>0 \\
0 \leq T E P \leq T E P_{\text {max }}
\end{gathered}
$$

Equations (11), (12) and (13) represent constrains more specific for the characteristics of each player.

\section{Case study}

The considered case study created is intended to be as realistic as possible. As previously mentioned, real data were used to carry out the training of the methods of obtaining prices, the scenario created has five different markets, which were given the market names Spot, Bilateral, Balancing (has two different sections) and Smart Grid. Negotiation rules have also been created that limit the option to sell and buy electricity in the market. The rules created were as follows:

- In the Spot market it is impossible to buy electricity; it can only be sold (according to the MIBEL - Iberian Market Operator rules for seller players [15])

- In the two balancing market sections only one of the actions can be carried out at each moment, either buy or sell electricity

- In the Bilateral and Smart Grid markets it is possible to sell and buy electricity during the same period

- Prices in the Bilateral and Smart Grid markets are directly influenced by the traded quantity

- In each market it is possible to buy only $10 \mathrm{MW}$ of electricity

- There is a generation amount of $10 \mathrm{MW}$

- No generation costs are considered in order to simplify the interpretation of the results 
- The sales quantity can reach $50 \mathrm{MW}$ in a single market

The assumptions defined already limit some of the considerations and restrictions presented, as is the case of equation (7) where the TEP is defined as the sum of the energy produced that in this case will have a maximum of 10, the value of equation (9) will be $0 €$, seen that the production is renewable and has no costs.

As already mentioned, the problem to be solved consists of two distinct objective functions that depend on each other. By the presented formulation were added the two objective functions in one with the use of a weighting factor $\lambda$. The values for $\lambda \in\{0.1,0.15,0.2,0.25,0.5,0.75,1\}$, the exposure to risk increases with increasing $\lambda$ value, which means that when $\lambda=$ 1 the option with the highest risk level will be performed. When analyzing the equation (1) can realize that when the $\lambda=1$ the equation $f 2$ annuls and will be given maximum importance to the equation $f 1$. Like this $\lambda$ values it will be possible to construct a plot of return vs risk that can be compared to a Pareto front

Considering the randomness of the resolution method (GA), 1000 simulations were performed for each level of risk, in each of the 1000 executions the algorithm can perform 10000 iterations, and the stopping criterion is defined by the solution variance at each iteration. All experiments were carried out on a computer compatible with 1 processor Intel ${ }^{\circledR}$ w $35653.2 \mathrm{GHz}$, with 4 Cores, 8 GB of RAM and operating system Windows 10 64 bites.

\section{RESUlts}

This section presents the results obtained from the application of the GA to the problem described above. First, the justification of the use of heuristics to solve the problem is presented in TABLE I.

TABLE I. RESULTS OF THE PROPOSED GA APPROACH AND OF THE DETERMINISTIC SOLUTION FOR $\lambda=1$

\begin{tabular}{|l|l|l|l|}
\hline \multirow{2}{*}{ Method } & \multicolumn{2}{|l|}{ Time (s) } & Objective function (€) \\
\cline { 2 - 4 } & Total & Mean & Maximum \\
\hline $\begin{array}{l}\text { Heuristic (GA) } \\
(1000 \text { runs) }\end{array}$ & 6834.32 & 6.83 & 2000.278 \\
\hline $\begin{array}{l}\text { MILP (Cplex } \\
\text { Solver) }\end{array}$ & 43710.68 & 2000.645575 \\
\hline
\end{tabular}

TABLE I compares the resolution using a Cplex deterministic technique and a heuristic technique the GA. As it can be observed the execution time of the Cplex is much higher than the one presented by GA. The deterministic approach takes about 12 hours to reach a solution, while the proposed GA approach needs only less than 7 seconds. This is even more significant given that the GA total value represents the 1000 simulations, less than 2 hours to execute 1000 times. In terms of objective function, as expected the Cplex clearly shows better results, although the GA presents a result with a difference of only $0.36 €$, which represents less than $0,1 \%$ difference.

TABLE II presents the results of GA application in all set of considered $\lambda$ values. As it is possible to observe, the results for the objective function equation (1) are present, as it was already from the 1000 simulations, the maximum, mean and the standard deviation (STD) value of the sample were calculated.

TABLE II . RESULTS FOR GA FOR DIFFERENT $\lambda$

\begin{tabular}{|l|l|l|l|}
\hline \multirow{2}{*}{$\lambda$ value } & \multicolumn{3}{|l|}{ Objective function $(\boldsymbol{€})$} \\
\cline { 2 - 4 } & Maximum & Mean & STD \\
\hline 0.1 & 105.0121 & 69.2479 & 8.875241 \\
\hline 0.15 & 175.232 & 139.008 & 15.97552 \\
\hline 0.2 & 249.9767 & 216.8515 & 21.6061 \\
\hline 0.25 & 355.1052 & 291.0924 & 36.82289 \\
\hline 0.5 & 880.7036 & 711.084 & 179.731 \\
\hline 0.75 & 1434.806 & 1259.568 & 195.253 \\
\hline 1 & 2000.278 & 1771.208 & 218.2482 \\
\hline
\end{tabular}

In Fig. 3, a graph is presented representing the average value of the equation (1) of the 1000 evaluations.

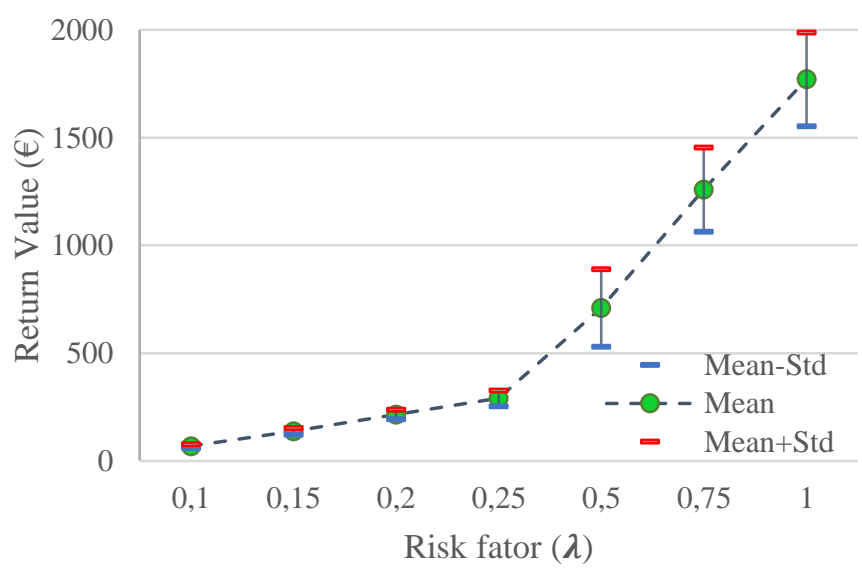

Fig. 3. Results for equation (1)

Fig. 3 shows the mean values of the 1000 simulations obtained from the equation of equation 1 as well as the STD. The points represented were chosen to perform the sensitivity to risk, at each point 1000 simulations were performed where the mean and the STD values were determined. By the figure it is visible that the value of the objective function increases with respect to the value of exposure to the risk, as well as the STD. It is important to analyze the STD because it indicates how the solutions approximate and dispersion of the mean value. In values where the risk exposure is lower, the STD value is also lower, this can be explained by the low values of the objective function.

Fig. 4 shows a plot of return versus risk value, the return is obtained by equation (2) and the risk is obtained by equation (3), each point represented in the graph represents different optimizations of equation (1) by applying the $\lambda$ factor.

Although the values relating to the $\lambda$ factor are not present in the plot, each point identified by a rhombus represents respectively a $\lambda$ value. As can be seen, the return and risk values increase with increasing lambda values. In the case of return the units of measure is $€$ 's, in risk there is no unit, it is considered adimensional. As can be seen, this representation can be 
compared to a representation of a Pareto function, although in this case it has been obtained by the application of the weighing factors ( $\lambda$ values). In Fig. 6 the different values of buying and sale of electricity in the different markets for the set of $\lambda$ are represented.

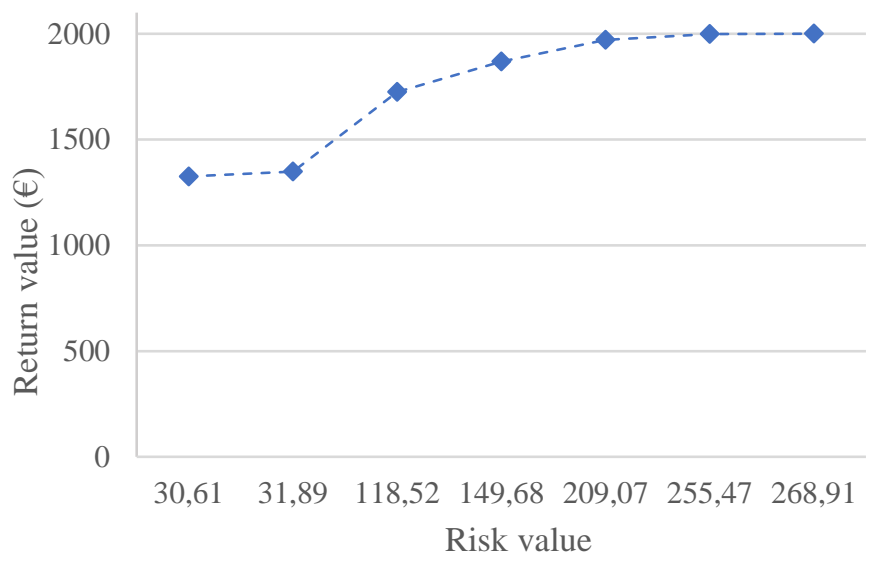

Fig. 4. Return vs. risk

Fig. 6 shows the allocation of the different optimizations for the different values of $\lambda$, the values represented differ with respect to the values of the variables for the maximum simulation found. The color scale indicates the exposure to risk, the heavier shades represent a higher return value, but also a higher risk value. The negative values represent electricity purchased, this representation was made to imply that the purchase of electricity contributes negatively to the objective function.

Fig. 5 shows the error value registered in all markets, as it can be observed, the market error of the balancing market session 2 is the highest with 0.25 , followed by SG, balancing market - session 1, bilateral contracts and day-ahead spot market. It is notable that with greater exposure to risk the allocation of electricity will tend to go to the markets where the risk is greater if the monetary point of view is more profitable.

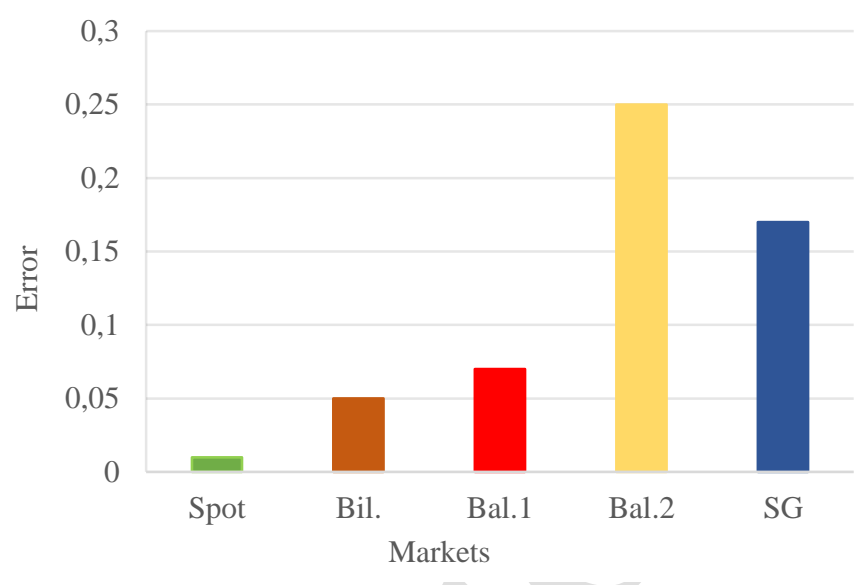

Fig. 5. Error values in different markets

Fig. 7 shows the variation of prices in the different markets, as can be observed and as already mentioned there are markets where the price of electricity is not constant varies with the quantity traded, as it happens with the markets Bilateral and SG.

By matching Fig. 5, Fig. 6, and Fig. 7 it is possible to understand how exposure to risk, error value and price of electricity influence the allocation of electricity. When the risk exposure is greater, larger amounts are allocated to markets where the error is greater as well. Analyzing the example of selling in SG market, with $\lambda=0.1$ the player has a small exposure to the risk, which indicates that the model allocates the sales to markets where the error is smaller, which is not the case. However, when $\lambda$ increases, sales are allocated in this market because the risk is less relevant. In this case, this market offers the possibility of more return from the electricity sale. In the case of the spot market, with a low $\lambda$ all electricity (production plus purchase) is allocated in this market, because it has the lower amount of error that implies lower risk. The volume sold in spot with a small level of risk does not reach the maximum amount that is possible to sell, because in order to achieve that maximum amount, there is a need to buy electricity as well. Since the electricity purchase risk is also considered in the proposed model, there are no purchases (or only small amounts), in markets where the risk is high, when $\lambda$ is low.

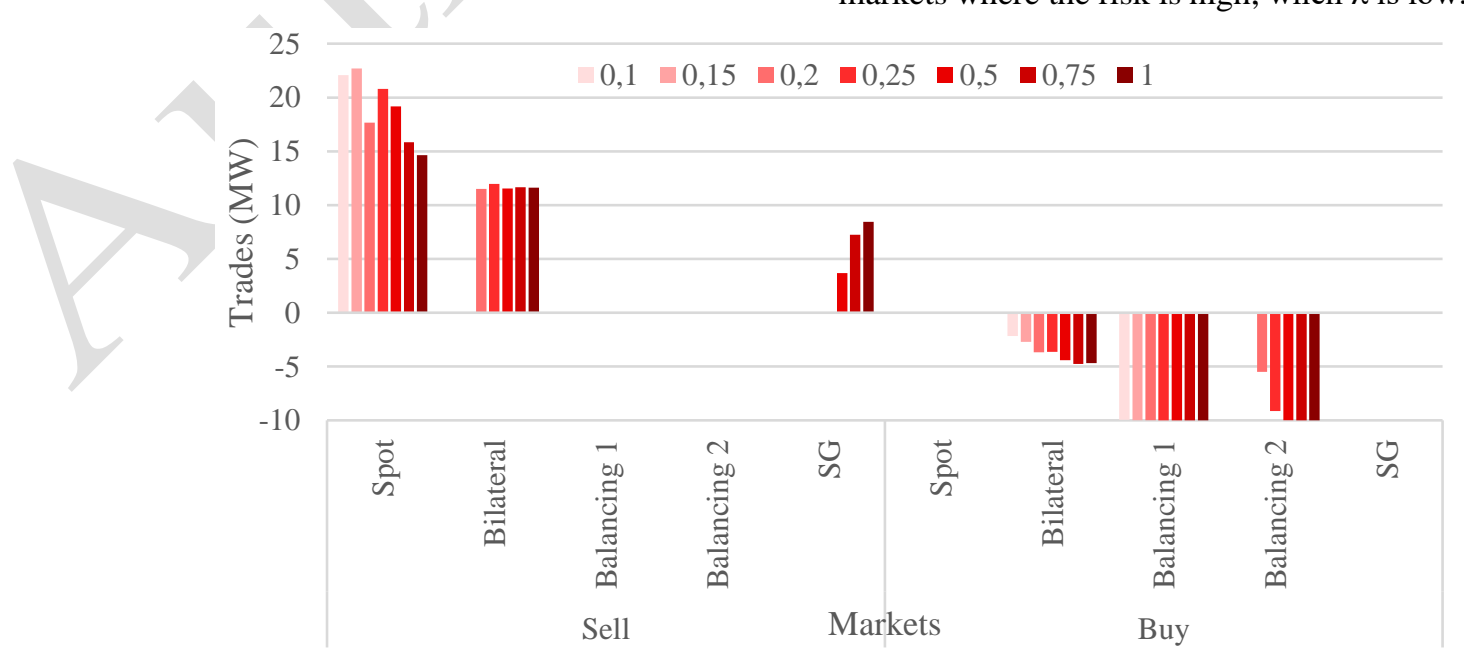

Fig. 6. Scheduling of sales and buying in different markets 


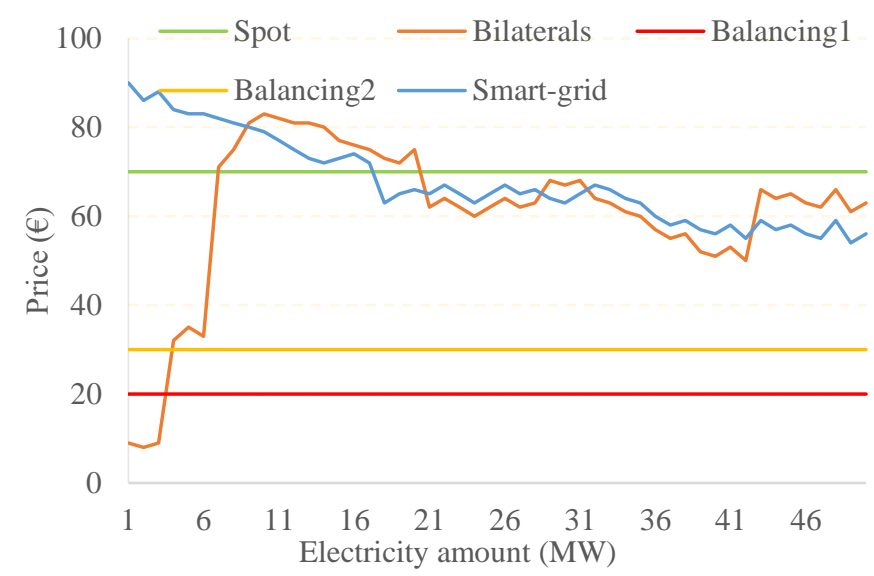

Fig. 7. Electricity Prices in different markets

TABLE III shows the results obtained regarding the mean value of iterations and the mean time obtained in solving the problem for the respective risk values.

TABLE III. ITERATIONS AND TIME RESULTS

\begin{tabular}{|c|c|c|c|c|}
\hline \multirow{2}{*}{$\lambda$ value } & \multicolumn{2}{|l|}{ Iterations } & \multicolumn{2}{|l|}{ Time } \\
\hline & Mean & STD & Mean & STD \\
\hline 0.1 & 2273.871 & 80.13818 & 6.189814 & 0.235532 \\
\hline 0.15 & 2341.223 & 85.22644 & 6.429584 & \begin{tabular}{|l|l|}
0.262409 \\
\end{tabular} \\
\hline 0.2 & 2371.491 & 114.2947 & 6.556798 & 0.336245 \\
\hline 0.25 & 2368.678 & 156.6457 & 6.605494 & 0.421316 \\
\hline 0.5 & 2316.408 & 275.7496 & 6.573109 & 0.773232 \\
\hline 0.75 & 2375.229 & 232.1405 & 6.769335 & 0.670076 \\
\hline 1 & 2393.342 & 209.7059 & 6.834315 & 0.614386 \\
\hline
\end{tabular}

As it is possible to observe the value of the exposure to the risk does not influence the number of iterations nor the execution time; this means that the GA behaves in the same way in the resolution of the problem. The value of the STD registers an increase with the increase of the exposure to the risk in the parameter of the iterations as well as in the execution time.

\section{CONCLUSION}

This paper proposes a model of multiple participation in electrical energy markets. The presented model allows the user to choose the level of risk that he wishes to incur. in this case it is possible to obtain the value of return and the value of risk incurred. At the time of the model the risk exposure model shows the amount of electricity purchased and sold in the different considered markets. The data used were collected from a real source (MIBEL) for a better approximation of reality. From the achieved results it is possible to verify that the methodology works correctly. respecting the imposed rules and obtaining positive values that represent profit for the user. The proposed GA approach was efficient in solving the problem. as for the execution time the GA clearly presents advantages in comparison to the exact resolution. presenting results much faster and very close to the exact results.
As previously discussed. the choice of $\lambda$ values is not easy and sometimes it may not present the expected results. In the case of the experiences performed with the set of $\lambda$ that are defined. it is not possible to obtain a prefect pareto front. but it is possible to perceive the relationship between the two objectives.

The future work authors intend to apply a different metaheuristic like Differential Evolution [16]. The application of multiobjective algorithms like NSGA-II [17] and SPEA2 [16], is also one of the future works, and will allow optimize the model without being necessary to use the weights. It is also proposed to account for the production risk in the case of renewable energies the forecast executed will always have an associated error.

\section{REFERENCES}

[1] D. E. Kvasov and M. S. Mukhametzhanov, "Metaheuristic vs. deterministic global optimization algorithms: The univariate case," Appl. Math. Comput., vol. 318, pp. 245-259, Feb. 2018.

[2] R. Faia, T. Pinto, and Z. Vale, "GA optimization technique for portfolio optimization of electricity market participation," in 2016 IEEE Symposium Series on Computational Intelligence, SSCI 2016, 2017.

[3] T. Pinto et al., "Adaptive Portfolio Optimization for Multiple Electricity Markets Participation,” Neural Networks Learn. Syst. IEEE Trans., vol. PP, no. 99, p. 1, 2015.

[4] H. Markowitz, "Portfolio Selection," J. Finance, vol. 7, no. 1, p. 77, Mar. 1952.

[5] R. Pérez Odeh, D. Watts, and M. Negrete-Pincetic, "Portfolio applications in electricity markets review: Private investor and manager perspective trends," Renew. Sustain. Energy Rev., , pp. 192-204, Jan. 2018.

[6] J. Kettunen, A. Salo, and D. W. Bunn, "Optimization of Electricity Retailer's Contract Portfolio Subject to Risk Preferences," IEEE Trans. Power Syst., vol. 25, no. 1, pp. 117-128, Feb. 2010.

[7] T.-J. Chang, S.-C. Yang, and K.-J. Chang, "Portfolio optimization problems in different risk measures using genetic algorithm," Expert Syst. Appl., vol. 36, no. 7, pp. 10529-10537, Sep. 2009.

[8] F. A. Roques, D. M. Newbery, and W. J. Nuttall, "Fuel mix diversification incentives in liberalized electricity markets: A Mean-Variance Portfolio theory approach,” Energy Econ., no. 4, pp. 1831-1849, Jul. 2008.

[9] M. Shafie-khah and J. P. S. Catalão, "A Stochastic Multi-Layer Agent-Based Model to Study Electricity Market Participants Behavior," IEEE Trans. Power Syst., vol. 30, no. 2, pp. 867-881, 2015.

[10] R. T. Marler and J. S. Arora, "The weighted sum method for multiobjective optimization: new insights," Struct. Multidiscip. Optim., vol. 41, no. 6, pp. 853-862, Jun. 2010.

[11] B. Milenova, "Fuzzy and neural approaches in engineering," Neural Networks, vol. 10, no. 9, pp. 1740-1741, Dec. 1997.

[12] M. Kumar, M. Husian, N. Upreti, and D. Gupta, "Genetic algorithm: review and application,” Int. J. Inf. Technol. Knowl. Manag., vol. 2, no. 2, pp. 451-454, 2010 .

[13] R. Faia, T. Pinto, and Z. Vale, "Dynamic Fuzzy Clustering Method for Decision Support in Electricity Markets Negotiation," ADCAIJ Adv. Distrib. Comput. Artif. Intell. J., vol. 5, no. 1, p. 23, Jul. 2016.

[14] T. Pinto, T. M. Sousa, and Z. Vale, "Dynamic artificial neural network for electricity market prices forecast," Intelligent Engineering Systems (INES), 2012 IEEE 16th International Conference on. pp. 311-316, 2012.

[15] MIBEL, "Mercado Iberico de Eletrecidade," 27-Feb-2007. [Online]. Available: http://www.mibel.com/index.php?lang=pt. [Accessed: 27Feb-2017].

[16] E. Zitzler, M. Laumanns, and L. Thiele, SPEA2: Improving the Strength Pareto Evolutionary Algorithm, vol. 103. 2001.

[17] K. Deb, A. Pratap, S. Agarwal, and T. Meyarivan, “A fast and elitist multiobjective genetic algorithm: NSGA-II," IEEE Trans. Evol. Comput., vol. 6, no. 2, pp. 182-197, Apr. 2002. 


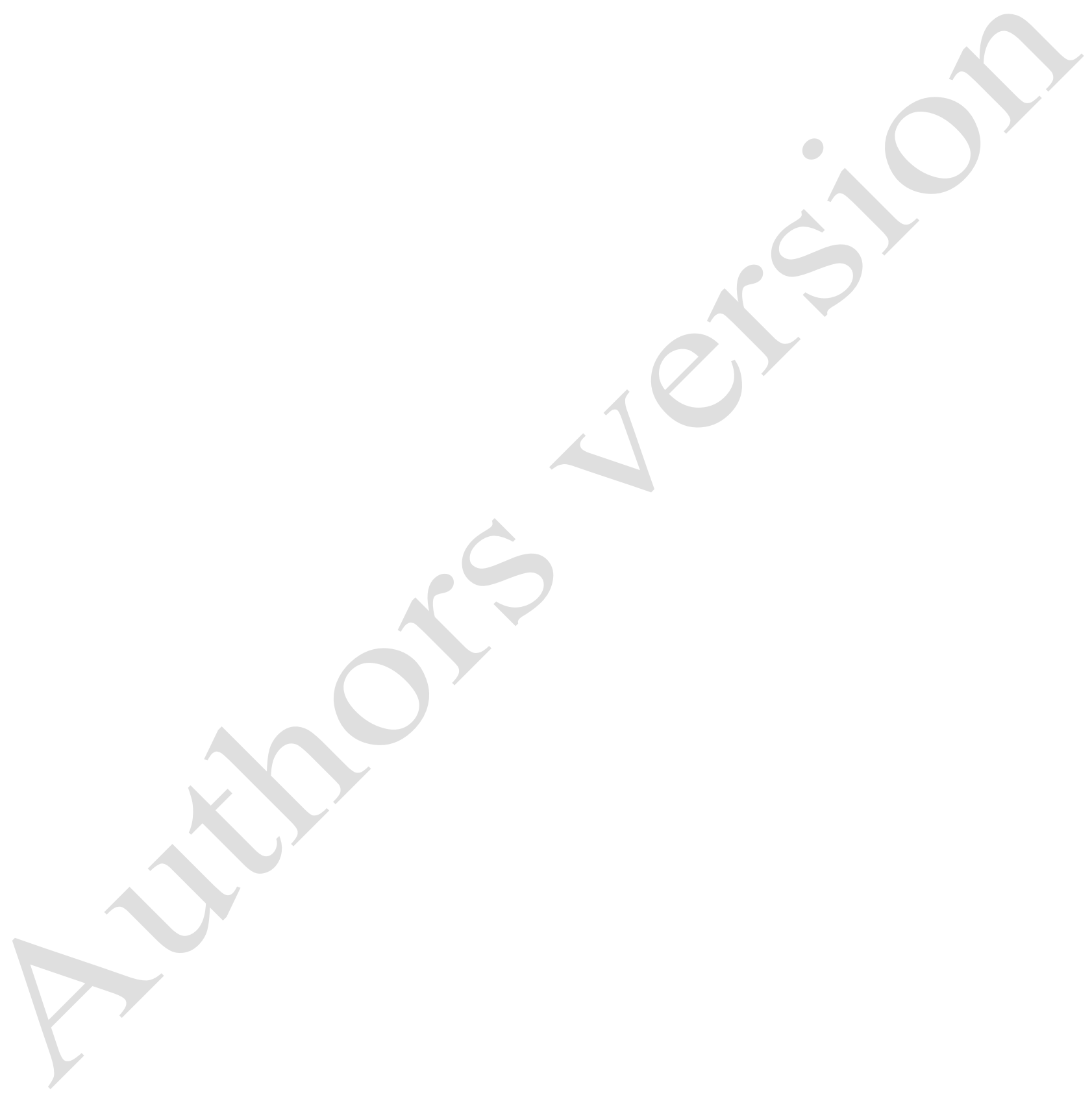

